\title{
11 Integration des Radverkehrs in zukünftige urbane Verkehrsstrukturen mit automatisierten und vernetzten Fahrzeugen
}

Lutz Eichholz, Detlef Kurth

1. Einleitung

2. Die Rahmenbedingungen für den Radverkehr 202

2.1 Rechtliche Einordnung 203

2.2 Fahrverhalten und Regelakzeptanz 203

2.3 Radfahrtypen 205

2.4 Unfälle von Radfahrenden 206

3. Rahmenbedingungen automatisierter und vernetzter Fahrzeuge 207

3.1 Fahrverhalten der automatisierten und vernetzten Fahrzeuge 207

3.2 Auswirkungen auf die Anzahl des ruhenden und fahrenden motorisierten
Individualverkehrs durch automatisierte und vernetzte Fahrzeuge

3.3 Flächenersparnis im Verkehrsraum durch automatisierte und vernetzte Fahrzeuge 209

4. Leitziele der deutschen Bundesregierung zum Radverkehr und automatisierten und vernetzten Fahrzeugen $\quad 211$

$\begin{array}{lll}4.1 & \text { Leitziele zum Radverkehr } & 211\end{array}$

4.2 Leitziele hinsichtlich automatisierten und vernetzten Fahrzeugen 211

5. Kriterien für die Drivability von automatisierten und vernetzten Fahrzeugen in der Interaktion mit Radfahrenden $\quad \mathbf{2 1 2}$

6. Eignung von Radverkehr-Führungsformen für avF: Mischverkehr oder getrennter Verkehr?

$\begin{array}{ll}\text { 7. Die Bedeutung von Knotenpunkten } & 216\end{array}$

$\begin{array}{llr}\text { 8. Fazit } & 217\end{array}$

$\begin{array}{lll}\text { 9. } & \text { Ausblick und Forschungsbedarf } & 218\end{array}$

$\begin{array}{ll}\text { Literatur } & 219\end{array}$

\footnotetext{
Lutz Eichholz

TU Kaiserslautern, Fachbereich Raum- und Umweltplanung

lutz.eichholz@ru.uni-kl.de

Detlef Kurth

TU Kaiserslautern, Fachbereich Raum- und Umweltplanung

detlef.kurth@ru.uni-kl.de
}

(C) Der/die Autor(en) 2021

M. Mitteregger et al. (Hrsg.), AVENUE21. Politische und

planerische Aspekte der automatisierten Mobilität,

https://doi.org/10.1007/978-3-662-63354-0_11 


\section{EINLEITUNG}

Eine der größten Herausforderungen für die Entwicklung von automatisierten und vernetzten Fahrzeugen (avF) ist es zur Zeit, Bewegungen von anderen Verkehrsteilnehmenden vorauszusehen (Kahn 2018, Mingels 2019). Der ehemalige CEO von Renault-Nissan-Mitsubishi, Carlos Ghosn, wird in Bezug auf Radverkehr und die fortschreitende Automatisierung von Kraftfahrzeugen (Kfz) mit den Worten zitiert: „One of the biggest problems is people with bicycles [...], [they] don't respect any rules usually [...] from time-to-time they behave like pedestrians and from time-to-time they behave like cars" (Reid 2018). Diese Aussage verdeutlicht die Konflikte, die durch ein Aufeinandertreffen zweier sowohl im Antrieb als auch in der Fahrweise stark abweichender Verkehrsmittel entstehen können.

Wie die kommenden Herausforderungen für den Radverkehr gelöst werden können, wenn in Städten automatisiertes Fahren stark zunimmt, untersucht die TU Kaiserslautern in dem interdisziplinären Forschungsprojekt „Konzepte zur Integration des Radverkehrs in zukünftige urbane Verkehrsstrukturen mit autonomen Fahrzeugen - RAD-AUTO-NOM“. Das Institut Mobilität und Verkehr (imove), das Fachgebiet Elektromobilität sowie der Lehrstuhl Stadtplanung erörtern gemeinsam die verkehrlichen, technischen und stadträumlichen Belange dieser Entwicklung. Von Juni 2019 bis Mai 2022 wird das Forschungsprojekt vom Bundesministerium für Verkehr und digitale Infrastruktur (BMVI) in Deutschland gefördert.

Im vorliegenden Beitrag wird auf die ersten Teilergebnisse der Arbeitspakete des Lehrstuhls Stadtplanung eingegangen. Aufgegriffen werden die stadträumlichen Chancen und Risiken, die sich für den städtischen Radverkehr im Wechselspiel mit avF ergeben. Aus Analysen des derzeitigen Verkehrsgeschehens sowie der Gestaltung des Verkehrs- und des öffentlichen Raums leiten wir ab, unter welchen Voraussetzungen automatisierte Fahrzeuge konfliktfrei integriert werden können. Zentrale Bedeutung hat die Frage, wie Städte geplant werden müssen, um in einer Zukunft mit avF und hohen Anteilen von Radverkehr lebenswerte Städte mit hoher Aufenthalts- und Städtebauqualität für alle zu erhalten.

\section{DIE RAHMENBEDINGUNGEN FÜR DEN RADVERKEHR}

Damit avF unfallfrei und nonstop fahren können, sollte der Verkehr vorhersehbar sein. Der „Edge Case“, eine eigentlich selten vorkommende Situation, beschreibt die intuitive Fahrweise von Radfahrenden im offenen Straßenraum, auf die avF nur schwer reagieren können.

In deutschen Metropolen ist zwischen 2002 und 2017 der Fahrradanteil an allen zurückgelegten Wegen von 9 auf 15 Prozent gestiegen, in Großstädten von 10 auf 14 Prozent (Nobis 2019: 21). Welche Folgen die Corona-Pandemie auf den Verkehr hat, ist noch schwer zu sagen. Dass sich die bestehenden Trends zu aktivem Verkehr verstärken und mehr Menschen den motorisierten Individualverkehr (MIV) nutzen, wird erwartet (vgl. Amelang 2020, Klein et al. 2020). Diese Trends unterstreichen, dass avF lernen müssen, konfliktfrei mit Radfahrenden zu interagieren.

Im folgenden Abschnitt wird erläutert, wie das Radfahren in Deutschland rechtlich geregelt ist, wie die Fahrweise einzuordnen ist, welche Radfahrertypen es gibt und welche Konflikte und 
Unfälle im derzeitigen Verkehrsgeschehen auftreten. Dabei differenzieren wir teilweise zwischen Fahrrad und Pedelec'. Andere Fahrradarten wie Lastenräder, Fahrräder mit Anhängern oder Tandems haben ähnliche Anforderungen wie klassische Fahrräder.

\section{$2.1 \quad$ RECHTLICHE EINORDNUNG}

Wenn keine eigene Infrastruktur für den Radverkehr vorhanden ist, müssen Radfahrende in Deutschland laut Straßenverkehrsordnung am rechten Fahrbahnrand fahren. In diesem Mischverkehr mit Kraftfahrzeugen sind 0,8 bis 1 Meter Abstand vom Fahrbahnrand angemessen. Der Abstand kann bei Gefahrensituationen (z. B. öffnende Autotüren) angepasst werden. Radfahrende dürfen andere Verkehrsteilnehmende links überholen, wenn ein ausreichender $\mathrm{Ab}$ stand eingehalten werden kann. Zusätzlich dürfen sie, wenn es die Situation gefahrlos zulässt, Kfz rechts überholen (ADFC 2018a). Einbahnstraßen werden für Radfahrende teilweise in Gegenrichtung geöffnet. In verkehrsberuhigten Bereichen ist es erlaubt, schneller als Schrittgeschwindigkeit zu fahren. Bis zum achten Lebensjahr müssen radfahrende Kinder Gehwege benutzen, bis zum zehnten Lebensjahr dürfen Kinder frei wählen, ob sie auf Radinfrastruktur oder dem Bürgersteig fahren (§ 2 Abs. 5 StVO).

Wenn eine Infrastruktur für den Radverkehr vorhanden ist, so ist zu unterscheiden, ob sie sich auf oder neben der Fahrbahn befindet. Schutz- sowie Radfahrstreifen werden auf der Fahrbahn angelegt. Schutzstreifen werden durch eine gestrichelte, Radfahrstreifen durch eine durchgezogene Linie gekennzeichnet. Schutzstreifen sind Teil der Fahrbahn und dürfen bei Bedarf von anderen Fahrzeugen genutzt werden, aber nur, wenn Radfahrende dabei nicht gefährdet werden. Radfahrstreifen dürfen von anderen Fahrzeugen nicht genutzt werden (ADFC 2018a). Darüber hinaus gibt es geschützte Radfahrstreifen (auch „Protected Bike Lane“ genannt) und eigenständige Radwege, die von Fahrspuren der Kfz getrennt sind (ADFC 2019). Radwege mit Nutzungspflicht müssen grundsätzlich mit einem Gebotsschild ausgewiesen werden und eine Mindestbreite von 1,6 Metern aufweisen (ADFC 2018a: 8; FGSV 2006: 84).

Beim Linksabbiegen haben Radfahrende zwei rechtlich konforme Möglichkeiten: Sie können rechtzeitig vor der Kreuzung ihre Spur (auch bei benutzungspflichtigen Radwegen) verlassen und von der Fahrbahnmitte aus abbiegen. Wenn vorhanden, können sie eine Radverkehrsführung benutzen, müssen dies aber nicht. Alternativ kann auch indirekt abgebogen werden, indem die Kreuzung erst geradeaus überfahren und dann vom rechten Fahrbahnrand aus gequert wird (ADFC 2018a; § 9 StVO).

\subsection{FAHRVERHALTEN UND REGELAKZEPTANZ}

Das Zitat „As a device the bike is so closely attuned to the body that it is almost as agile“ (Fleming 2017: 57; vgl. Beitrag 12 von Fleming in diesem Band) verdeutlicht die Fahrweise von Radfahrenden: Sie sind schnell, agil und wendig, haben in der Regel keine Fahrtrichtungsanzeiger oder Bremslichter und können sich sowohl im Kraftfahrzeug- als auch im Fußgängerverkehr bewegen. Zudem verhalten sie sich teilweise nicht regelkonform. In einer Umfrage gaben 62 Prozent der Radfahrenden an, sich nicht immer an alle Verkehrsregeln zu halten (BMVI 2019a: 29). Die häufig schlechte oder sogar fehlende Radinfrastruktur sind einer der Gründe für die schwankende Regelakzeptanz von Radfahrenden (Huemer/Eckhardt-Lieberam 2016, Schreiber/Beyer 2019).

1 Pedelecs („Pedal Electric Cycle“, E-Bike) sind Fahrräder mit Hilfsmotor, der sich nur bei aktivem Treten zuschaltet. Bis zu einer Geschwindigkeit von 25 km/h unterstützt der Elektromotor. Für Pedelecs gelten rechtlich die gleichen Regeln wie für Fahrräder ohne Motorunterstützung (ILS 2013: 9f.). 
Radfahrstreifen und Schutzstreifen, welche die Regelbreiten einhalten, werden von 86 bzw. 88 Prozent der Radfahrenden genutzt. Sind die Streifen schmaler als die Regelbreite, sinkt die Nutzungshäufigkeit um ungefähr 15 Prozent (Richter et al. 2019: 77). Lange Rotphasen führen zu mehr Rotlichtverstößen(Schwab 2019). Regelwidriges Linksfahren trittvorallem beiwichtigen Zielen auf, z. B. Einzelhandelsnutzung (Alrutz et al. 2009: 30).

Die hohe Agilität und Wendigkeit von Fahrrädern zeigt sich auch bei einem Vergleich der Bremswege von Kfz, E-Scooter und Fahrrad. Ausgehend davon, dass das Kfz im normalen Verkehrsablauf mit einer Geschwindigkeit von ca. 50 km/h und das Fahrrad maximal mit ca. 20 $\mathrm{km} / \mathrm{h}$ unterwegs ist, beträgt sein Anhalteweg im Vergleich zum Fahrrad geschwindigkeits- und massebedingt ungefähr das Fünffache. Auch ein E-Scooter mit gleicher Geschwindigkeit wie das Fahrrad benötigt einen längeren Anhalteweg (ADAC 2019):

- $\quad \mathrm{Kfz}(50 \mathrm{~km} / \mathrm{h})$ : Reaktionsweg $14 \mathrm{~m}+$ Bremsweg $14 \mathrm{~m}=28 \mathrm{~m}$

- E-Scooter (20 km/h): Reaktionsweg 5,5 m + Bremsweg $8 \mathrm{~m}=13,5 \mathrm{~m}$

- Fahrrad (20 km/h): Reaktionsweg 5,5 m + Bremsweg $4 \mathrm{~m}=9,5 \mathrm{~m}$

Es kann davon ausgegangen werden, dass der Reaktionsweg von avF niedriger ist als von fahrergeführten Fahrzeugen. Unabhängig davon wird der Anhalteweg bei $50 \mathrm{~km} / \mathrm{h}$ deutlich länger sein als bei Fahrrädern. Das Fahrrad ist also das Verkehrsmittel mit dem kürzesten Bremsweg. Dies trägt mit dazu bei, das unvorhersehbare Fahrmanöver praktiziert werden.

Zusammenfassend haben folgende Faktoren einen Einfluss auf das Fahrverhalten und die Regelakzeptanz von Radfahrenden: Radinfrastruktur, Komplexität des Verkehrsraums, subjektive Sicherheit, Radfahrertyp und der Gebietstyp (vgl. Abb. 1).

Abbildung 1: Faktoren für die Regelakzeptanz und Fahrweise von Radfahrenden

\section{Radinfrastruktur}

Radverkehrsanlagen müssen nicht nur sicher, sondern auch attraktiv und zielorientiert sein, um akzeptiert und benutzt zu werden.

\section{Komplexität des Verkehrsraums}

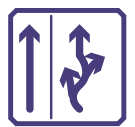

Intuitive Verständlichkeit vs. viele Auswahlmöglichkeiten und schwere Überschaubarkeit.

\section{Subjektive Sicherheit}

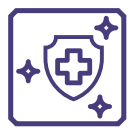

Als unsicher empfundene Infrastruktur wird seltener genutzt und verleitet dazu, unabhängig von rechtlichen Normen dort zu fahren, wo eine hohe Sicherheit empfunden wird (Bürgersteig statt schmaler Schutzstreifen, Fußgängerzone statt Straße).

\section{Radfahrertyp}

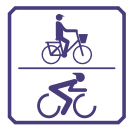

Unterschiedliche Radfahrtypen haben divergierende Fahrweisen: $v$ on langsam und regeltreu bis zu schnell, den direkten Weg fahrend, unabhängig von rechtlichen Vorgaben. 


\section{Gebietstyp}

Regelwidriges Linksfahren oder Queren von Straßen tritt bei Gebäuden,

die wichtige Ziele der Radfahrenden sind, häufiger auf.

Quelle: eigene Darstellung

\subsection{RADFAHRTYPEN}

Der Radfahrertyp ist ein wichtiger Indikator, um die Fahrweise und Anforderungen an die Infrastruktur und den öffentlichen Raum einschätzen zu können. In den Niederlanden gibt es eine Einteilung der Radfahrenden in sechs Gruppen: Merkmale sind die Fahrfähigkeiten, Motivation zum Radfahren sowie körperliche und berufliche Eigenschaften. Die sechs Gruppen können wie folgt beschrieben werden (Woolsgrove/Armstrong 2020):

- „Everyday Cyclist“ (Alltagsradlerln): Fahrten zur Arbeit oder zur Schule, direkte Wege, möchte ungestört fahren und so wenig wie möglich anhalten

- „Sporty Cyclist“ (Sportliche/r Radfahrerln): Fahrrad als Sportgerät (auch Mountainbike, Rennrad), fährt weite Strecken, fährt schnell;

- Recreational Cyclist“ (Freizeitradfahrerln): fährt aus Freude am Radfahren, fährt mit anderen, hält oft an Cafés oder Attraktionen an;

- Attentive Cyclist“ (Aufmerksame/r Radfahrerln): möchte sicher Fahrrad fahren, kennt die Verkehrsregeln und folgt diesen, möchte klare Verkehrszeichen und sichere Knotenpunkte;

- „Vulnerable Cyclist“ (Verletzliche/r Radfahrerln): meist Kinder, ältere Menschen und Menschen mit Einschränkungen, möchten sicher Fahrrad fahren und nicht von anderen Verkehrsteilnehmerlnnen (oder anderen Fahrrädern) überholt werden, die Infrastruktur muss Fehler verzeihen können;

- „Courier Cyclist“ (Berufliche/r Radfahrerln): möchte sein Ziel sehr schnell erreichen (Zeitdruck), braucht mehr Platz (Lastenrad, Anhänger).

Der Fahrradbeauftragte von Portland, Roger Geller, betrachtete darüber hinaus die Stresstoleranz von Radfahrenden im Straßenverkehr. Daraus ergeben sich vier Typen von Radfahrenden, die unterschiedliche Einstellungen der Bevölkerung zum Radfahren berücksichtigen:

- Furchtlose,

- Gewohnheitsfahrende,

- Interessierte,

- Nichtfahrende (NRVP 2019).

Diese „four types of cyclist“ sind heute eine anerkannte Klassifikation. Mit ihr verbunden ist jeweils die Aussage, welche Bedingungen für unterschiedliche Nutzergruppen erfüllt sein müssen, damit sie mit dem Rad mobil sein können (ADFC 2019, Dill 2015). Gellers Typologie wurde durch mehrere Studien an der Portland State University wissenschaftlich bestätigt und weiterentwickelt. Demnach sind 60 Prozent der Bevölkerung Interessierte, 5 Prozent Gewohnheitsfahrende und 2 Prozent Furchtlose. Die verbleibenden 33 Prozent sind Nichtfahrradfahrende (Geller 2009). 
Auf die Regelakzeptanz der einzelnen Typen wird bei vielen Klassifizierungen nicht explizit eingegangen. Bei den niederländischen Typen kann die Regelakzeptanz bei den „Everyday Cyclist“ und den "Courier Cyclist" als am niedrigsten angesehen werden. Bei der Typisierung von Geller indiziert schon der Name des „Furchtlosen“, dass seine Regelakzeptanz flexibel ist. VerkehrsökologInnen der TU Dresden ordnen die Gewohnheitsfahrenden in Bezug auf die Regelakzeptanz als niedrig ein (Francke et al. 2018: 10). Dies ist auf viele Radfahrtypen-Modelle übertragbar.

Inwiefern die Algorithmen von avF die unterschiedlichen Radfahrtypen in Zukunft erkennen können, ist schwer abzusehen. Da unterschiedliche Typen unterschiedliche Fahrweisen haben, könnten avF aber eventuell anhand dessen prognostizieren, welche Fahrweise (z. B. offensiv, defensiv, mit Regelakzeptanz) zu erwarten ist.

\subsection{UNFÄLLE VON RADFAHRENDEN}

\section{Unfallstatistik}

Laut Statistischem Bundesamt sind im Jahr 2018 in Deutschland insgesamt 88472 Unfälle mit Fahrrad- bzw. Pedelecbeteiligung geschehen. Zwischen 2017 und 2018 stieg die Anzahl der Unfälle von Radfahrenden in Deutschland um 11 Prozent (Destatis 2019). Noch höher liegen die Zahlen getöteter Rad- und Pedelecfahrender: Die Zahl ist von $382 \mathrm{im}$ Jahr 2017 auf $455 \mathrm{im}$ Jahr 2018 gestiegen (+16 Prozent; ebd.). Generell ist das Risiko, beim Radfahren zu sterben, laut Aussage des Unfallforschers Siegfried Brockmann, dreimal höher als beim Auto- und Motorradfahren. Die Wahrscheinlichkeit schwerer Verletzungen ist siebenmal höher (Burger 2019).

\section{Unfallgeschehen}

Dabei ereignen sich 90 Prozent der Unfälle von Radfahrenden innerorts (Destatis 2019). Außerorts haben Unfälle in der Regel deutlich schwerere Folgen: 40 Prozent dieser Unfälle ereignen sich mit tödlichen Folgen (BMVI 2012: 28). Ein Großteil der polizeilich erfassten Radunfälle geschieht an Knotenpunkten, beim Einbiegen oder Kreuzen der Fahrbahn (DLR 2015, Schreck 2016, GDV 2016). Bei Unfällen in Fahrtrichtung sind Ursachen für Radunfälle zu dichtes Auffahren, Überholen durch andere Verkehrsteilnehmende mit häufig zu geringem Abstand oder Behinderungen durch ruhenden Verkehr entlang von Radinfrastrukturen (LAB 2014, Richter et al. 2019, Tagesspiegel 2019, Schreiber/Beyer 2019, DVR 2019). Häufige Unfallursache ist außerdem die Missachtung der Vorfahrt oder die Fehleinschätzung der Geschwindigkeit durch andere VerkehrsteilnehmerInnen (GDV 2015). Wenn es zur Kollision kommt, ist in 75 Prozent der Fälle der Pkw der Hauptunfallverursacher (Destatis 2019).

\section{Unfälle mit dem ruhenden Verkehr}

Eine Studie des Gesamtverbands der Deutschen Versicherungswirtschaft e.V. (GDV) aus dem Jahr 2019 hat ergeben, dass neben markierten Radinfrastrukturen (Radfahrstreifen und Schutzstreifen) parkende Fahrzeuge einen erheblichen Einfluss auf die Anzahl von Unfällen mit Fahrrädern haben. Der Studie zufolge geschehen 65 Prozent der Unfälle auf Radfahr- bzw. Schutzstreifen durch sich öffnende Pkw-Türen (sog. Dooring-Unfälle; Schreiber/Beyer 2019): Die Tatsache, dass 16 Prozent der Autofahrenden beim Türöffnen keinen Schulterblick machen, erhöht für vorbeifahrende RadlerInnen das Risiko für Unfälle stark (DVR 2019). In diesen Parkbereichen ist die Unfallhäufigkeit ungefähr viermal so hoch wie ohne die Parksituation.

Aber auch ohne Unfälle stellen parkende Fahrzeuge eine erhebliche Behinderung für Radfahrende dar, insbesondere wenn sie auf Radwegen - und damit falsch - abgestellt sind. Solche Beeinträchtigungen behindern einen effizienten und sicheren Radverkehr: Laut einer Studie sind 40 Prozent von 40000 ausgewerteten Radfahrenden auf Schutzstreifen davon betroffen. Bei einer Erhebung auf 35 Radfahrstreifen und 47 Schutzstreifen wurden zahlreiche regelwidrige Parkvorgänge von Pkw festgestellt: Auf Schutzstreifen wurden pro Kilometer und Stunde 6,1 parkende und 
10,1 haltende Pkw gemessen, auf Radfahrstreifen 1,1 parkende und 4,5 haltende Pkw pro Kilometer und Stunde gemessen (Richter et al. 2019). Insgesamt wurde von der GDV festgestellt, dass fast jeder fünfte Unfall von Radfahrenden im Zusammenhang mit dem Parken steht (GDV 2020).

\section{RAHMENBEDINGUNGEN AUTOMATISIERTER UND VERNETZTER FAHRZEUGE}

Wann avF in Deutschland eingeführt werden können, ist schwer zu prognostizieren. Die Aussagen zur technischen Machbarkeit von IT- und von Automobilunternehmen sind sehr uneinheitlich und von der Konkurrenzsituation beeinflusst.

In einer Anfang 2019 vom Fraunhofer-Institut für System- und Innovationsforschung veröffentlichten Studie wird davon ausgegangen, dass sich neue Automatisierungstechnologien anfangs stärker im öffentlichen Personennahverkehr (ÖPNV) und bei Lkw verbreiten und anfangs vor allem höherpreisige Kfz damit ausgestattet werden. Unabhängig vom jeweiligen Verkehrsmittel geht die Studie davon aus, dass auch im Jahr 2050 die automatisierten Fahrzeuge ab Level $4^{2}$ noch in der Minderzahl sind (ISI 2019: 109-119). Neben der deutschlandweiten Diffusion von avF wird davon ausgegangen, dass ihre stadt- und verkehrsräumliche Verbreitung sehr unterschiedlich ausfallen wird: AvF werden in komplexen Umgebungen nicht oder nur langsam fahren können (vgl. Soteropoulos et al. 2020; Mitteregger et al. 2020: 81-84).

\subsection{FAHRVERHALTEN DER AUTOMATISIERTEN UND VERNETZTEN FAHRZEUGE}

Automatisierte und vernetzte Fahrzeuge bieten die Chance, dass die geltenden Verkehrsregeln vollständig durchgesetzt werden (Beckmann/Sammer 2016: 4; Rothfuchs/Engler 2018: 569). Wie die Fahrzeuge bei Gefahr einer Kollision reagieren sollen, ist ethisch umstritten und nicht abschließend festgelegt (vgl. Bonnefon et al. 2016). Bezüglich der Sicherheit von Radfahrenden ist zu berücksichtigen, wie avF in Bezug auf Situationen, in denen sie zwischen dem eigenen Schutz oder Fremdschutz entscheiden müssen, programmiert werden. Hierbei können neue Konfliktpunkte und Unfallursachen für Fahrräder entstehen, wenn das avF beispielsweise wegen eines entgegenkommenden Lkw ausweichen muss und auf den Schutz- oder Radfahrstreifen fährt (Awad et al. 2018).

Im Forschungsprojekt wird davon ausgegangen, dass avF in Deutschland folgende Fahreigenschaften haben werden:

- Geschwindigkeitsbegrenzungen werden nicht überschritten, dies gilt auch für Überholvorgänge.

- Die rechtlich verpflichtenden Sicherheitsabstände zu anderen Verkehrsteilnehmenden werden immer eingehalten. Ist dies nicht möglich, stoppt das Fahrzeug oder verlangsamt die Geschwindigkeit, bis die Abstände eingehalten werden können.

2 Im Beitrag ist mit Level die jeweilige Automatisierungsstufe nach SAE-Norm J3016 gemeint (SAE International 2018). 
- Ausnahmen von Verkehrsregeln gibt es nur, wenn durch die Regeleinhaltung Unfälle entstehen. Bei kritischen Situationen besteht die höchste Priorität darin, anzuhalten.

- AvF können nicht mit allen anderen Verkehrsteilnehmenden digital kommunizieren. Sie müssen andere Verkehrsteilnehmende und Hindernisse visuell wahrnehmen.

- Die in avF verwendeten Algorithmen haben das Ziel, die Sicherheit der eigenen FahrzeuginsassInnen gleichwertig mit der Sicherheit aller Verkehrsteilnehmenden zu werten.

Gerade aufgrund der gesetzlichen Überholabstände werden avF eine defensive Fahrweise praktizieren. Seit der StVO-Novelle 2020 gilt in Deutschland ein Mindestabstand von 1,5 Metern beim innerörtlichen Überholen von Radfahrenden ( 5 Abs. 4 StVO). Ebenso ist die Einführung eines neuen Verkehrsschildes beschlossen, welches an bestimmten Stellen Autos verbietet, Radfahrende zu überholen (BMVI 2020).

\subsection{AUSWIRKUNGEN AUF DIE ANZAHL DES RUHENDEN UND FAHRENDEN MOTO- RISIERTEN INDIVIDUALVERKEHRS DURCH AUTOMATISIERTE UND VERNETZ- TE FAHRZEUGE}

Inwiefern der öffentliche Raum sowie der Straßenraum im „Jahrhundert des sehenden Fahrzeuges“ (Dickmanns 2020) umgestaltet werden kann, hängt im hohen Maß davon ab, ob künftig mit steigendem oder verringertem motorisiertem Individualverkehr (MIV) gerechnet werden muss. Viele Studien, die sich mit dem Thema beschäftigen, gehen derzeit davon aus, dass der Individualverkehr mit steigender Automatisierung zunimmt (Heinrichs 2015, 2017; Botello et al. 2019; Millard-Ball 2019; Lee et al. 2019; Weert/Ruhrort 2019; Mitteregger et al. 2020: 37). Gründe dafür sind eine höhere Nachfrage durch neu akquirierte Nutzergruppen (z. B. Menschen mit Fahrbeeinträchtigungen), eine erhöhte Attraktivität des Individualverkehrs und in Zukunft auch Leerfahrten (Heinrichs 2015, 2017; Botello et al. 2019). Die verstärkte Skepsis gegenüber geteilten Verkehrsmitteln bzw. ÖPNV durch die Covid-19-Pandemie 2020 könnte mittelfristig zu einer gesteigerten MIV-Nutzung führen - abhängig von der künftigen Entwicklung des Virus sowie der damit bestehenden Möglichkeiten zur Eindämmung.

Bei einem Verkehr, der ausschließlich aus automatisierten und vernetzten Fahrzeugen besteht, hätten die städtischen Verkehrsräume theoretisch höhere Kapazitäten als bei fahrergeführten Fahrzeugen. Gründe sind schnellere Reaktionszeiten beim Anfahren und das Fahren im Verbund bzw. das verdichtete Fahren mit wenig Abständen zwischen einzelnen Fahrzeugen (Heinrichs 2015; Beckmann/Sammer 2016: 4; Rothfuchs/Engler 2018). Ein Nachtteil ist, das avF diese Vorteile nur anwenden können, wenn sie nicht mit fahrergeführten Fahrzeugen und anderen Verkehrsteilnehmenden interagieren müssen. Dafür müsste großflächig neue Infrastruktur angelegt werden. Eine Alternative wäre, in bestimmten Bereichen andere Verkehrsteilnehmende auszuschließen, was eine Rückkehr zu den Prinzipien der autogerechten Stadt bedeuten würde (vgl. Dangschat 2018).

Der Flächenverbrauch der avF für den ruhenden Verkehr könnte sich stark reduzieren - wie stark, ist schwer zu prognostizieren. Studien, die einen theoretischen Wert von 100 Prozent avF angenommen haben, gehen von Einsparungen bis zu 90 Prozent des ruhenden Verkehrs aus, wenn avF von mehreren NutzerInnen zeitgleich verwendet werden (Heinrichs 2015; Rothfuchs/ Engler 2018; Ritz 2018; Lemmer 2019: 26-29; Millard-Ball 2019: 5; Mitteregger et al. 2020: 74). Andere Forschende rechnen mit deutlich weniger Einsparungen (Skinner/Bidwell 2016, Alessandrini et al. 2015). Bei den unterschiedlichen Studien ist zu beachten, dass ein signifikanter 
Rückgang des ruhenden Verkehrs erst prognostiziert wird, wenn es hohe Anteile von Level-4und -5-Fahrzeugen mit hohen Besetzungsraten gibt.

Auch wenn sich die Annahmen zu Kosten und Flächenersparnis durch avF auf eine relativ ferne Zukunft beziehen, sind Städte bereits jetzt gefordert, zukunftsfähig zu planen. Sinnvoll ist es, Maßnahmen zu entwickeln, die aktiven Verkehr, Elektrokleinstfahrzeuge und ÖPNV fördern und dabei die steigenden Anteile von avF mit in Betracht ziehen (ISI 2019: 157). Zielbringende Maßnahmen wären die Bereitstellung von mehr und qualitativ hochwertigeren Flächen für aktive Mobilität, Mautsysteme für Kern- oder Innenstädte, das Ausweisen von Straßen- oder Fahrspuren, die Pkw mit mehr Insassen bevorzugen, und der Ausbau des ÖPNV, insbesondere auch des ÖPNV on demand. Eine große Chance von avF liegt darin, dass dessen Systeme sich zuerst in hochpreisigen Pkw, Bussen und Lkw durchsetzen (ebd.: 109-119). In dieser Übergangszeit kann der ÖPNV eine deutlich höhere Nutzerfreundlichkeit und Kosteneffizienz entwickeln. Gründe sind, dass deutlich weniger Personal benötigt wird und sich On-Demand-Systeme bei einem automatisiert fahrenden ÖPNV leichter einführen lassen. Zusätzlich können bestehende Lücken im ÖPNV-Netz durch On-Demand-Dienste geschlossen werden, wobei die Abhängigkeit vom motorisierten Individualverkehr, unabhängig ob automatisiert oder fahrergeführt, verringert werden kann.

\subsection{FLÄCHENERSPARNIS IM VERKEHRSRAUM DURCH AUTOMATISIERTE UND VERNETZTE FAHRZEUGE}

Wie im vorherigen Abschnitt geschildert, können hauptsächlich Flächen, die heute für den ruhenden Verkehr genutzt werden, durch avF eingespart werden. Dies kann nur geschehen, wenn künftig Fahrten vermehrt geteilt werden. Um darzustellen, wie sich der Verkehrsraum verändern könnte, ist die derzeitige Raumaufteilung zu ermitteln.

Es gibt nur wenige Erhebungen über die Aufteilung des Straßenraums in Städten. Im Jahr 2014 wurde in Berlin untersucht, welche Anteile an den Verkehrsflächen für die unterschiedlichen Verkehrsarten bereitgestellt werden. Das Ergebnis zeigt, dass 58 Prozent der Verkehrsflächen für Autos reserviert sind, 19 Prozent davon für parkende Fahrzeuge; 33 Prozent der Fläche nehmen Fußwege ein, der eigenständige Radverkehr verfügt nur über 3 Prozent der Verkehrsfläche (Agentur für clevere Städte 2014). In Kopenhagen untersuchten die Blogger von copenhagenize.com, wie auf dem Hans Christian Andersen Boulevard der Verkehrsraum aufgeteilt ist. Das Ergebnis zeigt, dass der größte Teil der Flächen dem motorisierten Verkehr zur Verfügung gestellt wird (54 Prozent, davon 12 Prozent für Parkplätze am Straßenrand), Fußgängerlnnen nutzen 26 Prozent und Radfahrende 7 Prozent der Flächen (Copenhagenize.com 2017). Eigene Stichproben, die im Forschungsprojekt anhand von Online-Stadtplänen durchgeführt wurden, führen in sieben StraBen und an vier Kreuzungen in Kaiserslautern, Mannheim und Karlsruhe zu ähnlichen Ergebnissen.

In der Richtlinie für die Anlage von Stadtstraßen (RASt 06) sind in 59 von 97 empfohlenen StraBenquerschnitten Flächen für den ruhenden Verkehr enthalten. Einzig für Wohnwege und anbaufreie Straßen werden in den Beispielquerschnitten keine Flächen für den ruhenden Verkehr ausgewiesen. Im Mittel aus allen Beispielquerschnitten sind 14 Prozent der Verkehrsflächen für den ruhenden Verkehr (Parken und Abstandsflächen) reserviert (eigene Auswertung der RASt 06 nach FGSV 2006: 36-62, vgl. Tab. 1).

3 Die Richtlinien für die Anlage von Stadtstraßen (RASt 06) sind ein in Deutschland gültiges technisches Regelwerk. Sie werden von der Forschungsgesellschaft für Straßen- und Verkehrswesen in Köln herausgegeben und werden in allen Bundesländern zur Anwendung empfohlen. 
Tabelle 1: Empfohlene Straßenquerschnitte nach RASt 06 (FGSV 2006)

\begin{tabular}{|c|c|c|}
\hline & Fahrbahn & Parken \\
\hline Wohnstraße & $4-6,5 \mathrm{~m}$ & $\begin{array}{l}\text { Teilweise ohne (4 von 9) } \\
\text { 14-30\% der Querschnittsbreite }\end{array}$ \\
\hline Sammelstraße & $5,5-7,5 \mathrm{~m}$ & $\begin{array}{l}\text { Teilweise ohne (3 von 8) } \\
\text { 23-31\% der Querschnittsbreite }\end{array}$ \\
\hline Quartierstraße & $5,5-7,5 \mathrm{~m}$ & $\begin{array}{c}\text { Teilweise ohne (1 von } 5) \\
19-26 \% \text { der Querschnittsbreite }\end{array}$ \\
\hline Örtliche Einfahrtstraße & $6,5-7,5 \mathrm{~m}$ & $\begin{array}{c}\text { Teilweise ohne (2 von 7) } \\
\text { 13-23\% der Querschnittsbreite }\end{array}$ \\
\hline Hauptgeschäftsstraße & $6-13 \mathrm{~m}$ & $\begin{array}{l}\text { Teilweise ohne (4 von } 13) \\
11-21 \% \text { der Querschnittsbreite }\end{array}$ \\
\hline
\end{tabular}

Quelle: FGSV (2006), eigene Darstellung und Auswertung nach Louen (2020)

Basierend auf den wenigen Erhebungen und der Auswertung der RASt 06 ist davon auszugehen, dass in deutschen Städten ungefähr 15 Prozent der Verkehrsflächen für den ruhenden Verkehr zur Verfügung gestellt werden (vgl. Abb. 2). Der Anteil schwankt dabei je nach Straßentyp stark. Straßen ohne Flächen für das Parken sind kaum vorhanden.

Abbildung 2: Aufteilung ausgewählter großstädtischer Straßenräume nach Verkehrsteilnehmenden

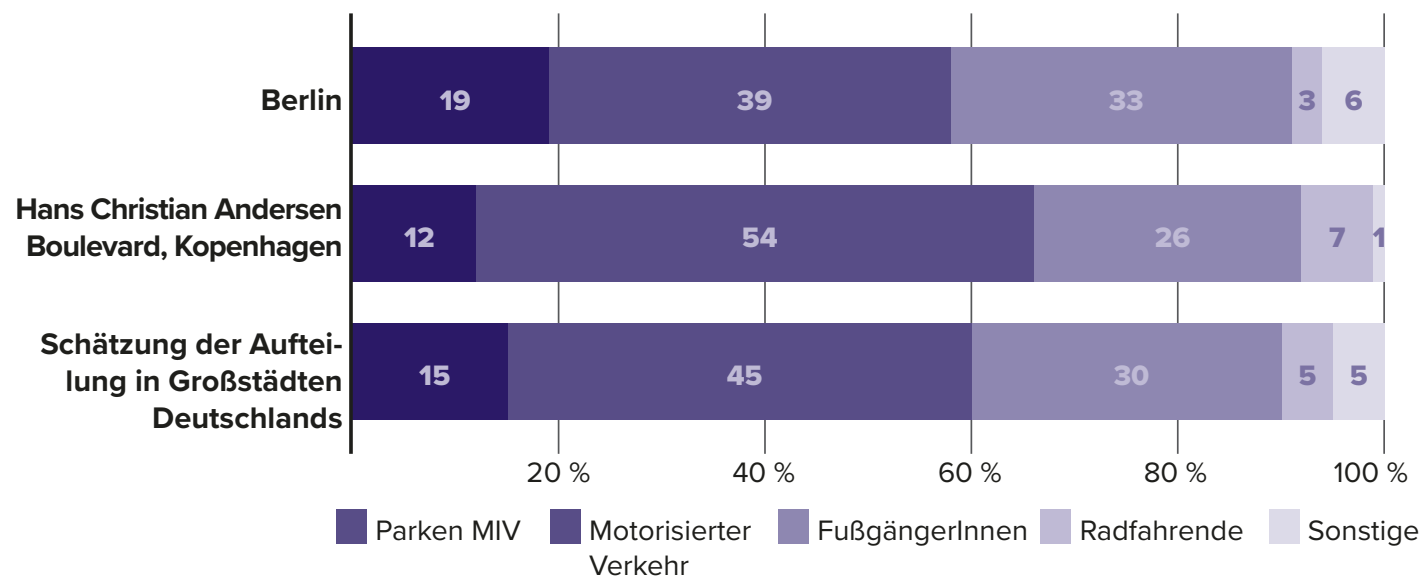

Quelle: Berlin - Agentur für clevere Städte (2014); Kopenhagen - Copenhagenize.com (2017); eigene Darstellung (Schätzung der Aufteilung in Großstädten Deutschland)

Die Flächen für ruhende Pkw könnten bei avF, die als Ride-Sharing-Systeme genutzt werden und somit fast ständig in Bewegung sind, eingespart werden. Dass dieses Szenario in naher Zukunft eintritt, ist aber nicht wahrscheinlich. Aus diesem Grund ist es zielführend, davon auszugehen, dass sich mit der steigenden Einführung von avF zunächst wenig an dem Bedarf von Flächen für den ruhenden und fließenden Verkehr ändert. Ein auf die Vermeidung von parkenden Fahrzeugen ausgerichtetes innerstädtisches Verkehrskonzept kann bereits zum jetzigen Zeitpunkt anfangen, Straßenprofile mit höherer Aufenthaltsqualität und einer gerechten 
Flächenverteilung zwischen ÖV (öffentlichem Verkehr), MIV und aktiver Mobilität zu fördern. Damit kann neben Flächen für Radverkehrinfrastruktur auch Platz für dezentrale, hochwertige Freiräume geschaffen werden. Statt den Platz nur dem MIV zur Verfügung zu stellen, können Sport, Erholungs-, Spiel- und Grünflächen sowie in innerstädtischen Gebieten größere Außenbereiche in der Gastronomie ermöglicht werden.

\section{LEITZIELE DER DEUTSCHEN BUNDESREGIERUNG ZUM RADVERKEHR UND AUTOMATISIERTEN UND VERNETZTEN FAHRZEUGEN}

\subsection{LEITZIELE ZUM RADVERKEHR}

Die Förderung des Radverkehrs leistet einen wichtigen Beitrag zur Erreichung von Umweltzielen sowie zur Lösung von Verkehrsproblemen in Innenstädten. Viele europäische Städte verzeichnen eine Zunahme des Radverkehrsanteiles (Schreiber/Beyer 2019), der auch in Deutschland gesteigert werden soll. Der Nationale Radverkehrsplan (NRVP) 2020 nennt als zentrale Aspekte sichere Infrastrukturen sowie ein hohes Sicherheitsgefühl der Radfahrenden. Der NRVP nennt acht Leitziele: Viele davon verfolgen eine deutliche Steigerung des Radverkehrsanteiles aller Altersklassen und Nutzungsformen (Freizeit, Beruf, Lastenverkehr). Ein weiteres Ziel fordert ein lückenloses Radverkehrsnetz mit konfliktarmen Infrastrukturen, um das subjektive und objektive Sicherheitsempfinden zu steigern sowie die Aufenthaltsqualität in den Städten zu erhöhen. Um diese Ziele zu erreichen, soll Digitalisierung sowie eine effektive Planung genutzt werden (BMVI 2019b).

\subsection{LEITZIELE HINSICHTLICH AUTOMATISIERTEN UND VERNETZTEN FAHRZEUGEN}

Die Strategie automatisiertes und vernetztes Fahren der Bundesregierung hat zum Ziel, Deutschland als Leitanbieter und Leitmarkt zu etablieren. Es soll frühzeitig ermöglicht werden, avF im Test- und Regelbetrieb zu erproben. Die Bundesregierung erhofft sich von automatisierter und vernetzter Mobilität (avM) eine Steigerung von Effizienz und Sicherheit bei weniger Emissionen sowie die Stärkung Deutschlands als Innovations- und Wirtschaftsstandort. Um dies zu ermöglichen, sind 2015 folgende Leitziele festgelegt worden: „die Anpassung des nationalen Rechtsrahmens, insbesondere die Änderung des Straßenverkehrsgesetzes, die Verabschiedung eines Maßnahmenplans zur Schaffung von Ethik-Regeln für Fahrcomputer, die Einrichtung und Koordinierung von Testfeldern für das automatisierte und vernetzte Fahren im Realverkehr, die Unterstützung der Forschung und Entwicklung von avF-Lösungen von der Grundlagen- bis zur Anwendungsforschung, die aktive Gestaltung von Regelungen und Standards in Gremien auf europäischer und internationaler Ebene“" (BMVI 2015).

Um negative Auswirkungen von avM zu vermeiden, fordert das Beratungsgremium der Bundesregierung, die Nationale Plattform Zukunft der Mobilität, dass im Zuge automatisierter Mobilität der MIV nicht ansteigen sollte. Als Gründe für mögliche Steigerungseffekte beim MIV werden u. a. Leerfahrten und Verlagerungseffekte von anderen Verkehrsarten wie dem Radverkehr und dem ÖPNV genannt (NPM 2019: 5).

Die Bundesregierung hat als Ziel formuliert, dass Deutschland das erste Land weltweit sein wird, das autonome Kraftfahrzeuge im Regelbetrieb und im gesamten nationalen Geltungsbereich erlaubt (Seibert 2020). 


\section{KRITERIEN FÜR DIE DRIVABILITY VON AUTOMATISIERTEN UND VERNETZTEN FAHRZEUGEN IN DER INTERAKTION MIT RADFAHRENDEN}

Ob automatisierte Fahrfunktionen genutzt werden können, hängt stark von der jeweiligen Umgebung, in der sich das Fahrzeug bewegt, ab (Soteropoulos et al. 2020, Mitteregger et al. 2020, SAE International 2018). Welche Kriterien in Bezug auf Radfahrende die "drivability“ (Fahrbarkeit) von avF ermöglichen, wurde aus den vorherigen Abschnitten hergeleitet.

Folgende Kriterien ergeben sich nach Abwägung des Fahrverhaltens von Radfahrenden und der daraus folgenden Ansprüche an die Infrastruktur: Straßenbreite, Art der Radinfrastruktur, Geschwindigkeit der Verkehrsteilnehmenden, die Möglichkeit zur Einschätzung der Fahrweise Radfahrender (freie Sichtachsen), Art des Radfahrenden sowie Anzahl und Art der Verkehrsteilnehmenden (vgl. Abb. 3).

Die Straßenbreite, die Anzahl von Radfahrenden, die Art der Radinfrastruktur und die Geschwindigkeit des Verkehrs haben dabei den größten Einfluss auf ein gelungenes Miteinander von avF und Radfahrenden. Bei einer angepassten Geschwindigkeit von 20 bis $30 \mathrm{~km} / \mathrm{h}$ können Sicherheitsabstände deutlich geringer ausfallen, im Weiteren müssen Sichtachsen über kürzere Streckenabschnitte gewährleistet sein. Nach RASt 06 halbieren sich die freizu-

Abbildung 3: Übersicht der Kriterien für die Drivability von automatisierten und vernetzten Fahrzeugen in der Interaktion mit Radfahrenden

\section{Straßenbreite}

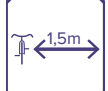

Sicherheitsabstände und Einhalten des

Überhollabstandes von 1,5 Metern möglich

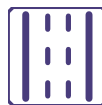

Anzahl der Spuren

Einschätzung der Fahrweise Radfahrender möglich

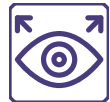

Freie Sicht (Behinderung durch parkende $\mathrm{Kfz}$, Vegetation, Infrastruktur)

Regeltreue (Vorhandensein und Qualität von

Radinfrastruktur, Gebietstyp, Radfahrertyp)

\section{Radinfrastruktur}

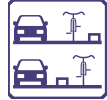

国正

Keine, Mischverkehr

(Schutzstreifen,

Radfahrstreifen),

überwindbare und

unüberwindbare Trennung

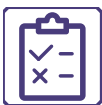

Qualität der Infrastruktur

(Regelbreite erfüllt,

Belagsqualität)
Geschwindigkeit der

Verkehrsteilnehmenden

政正 Bremswege

Anzahl und Art der Verkehrsteilnehmenden

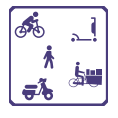

Radfahrende, Elektrokleinstfahrzeuge, Warenlieferanten, Zufußgehende

Fahrweise (defensiv, offensiv) bzw. Radtyp 
haltenden Bereiche im Seitenraum, wenn die Geschwindigkeit von 50 auf $30 \mathrm{~km} / \mathrm{h}$ reduziert wird (FGSV 2006: 88).

Eine geringe Straßenbreite bedeutet für avF, dass sie Radfahrende nicht überholen können und die Geschwindigkeit entsprechend anpassen müssen. Die Fahrweise von Radfahrenden wird dabei zum einen von der Infrastruktur, aber auch von der Umgebung geprägt. Liegen am Straßenrand Wohngebäude oder Gebäude, die wichtige Ziele von Radfahrenden sind, steigt die Wahrscheinlichkeit, dass unvorhersehbare Fahrmanöver durchgeführt werden. Das macht es für avF schwer, konfliktfrei und regelkonform zu fahren.

Die Heterogenität der Straßenräume stellt avF vor unterschiedlich komplexe Herausforderungen. Wenn viele Radfahrende mit unterschiedlichen Fahrweisen gleichzeitig unterwegs sind und wegen fehlender Sichtachsen im engen Straßenraum nicht immer zu detektieren sind, wird die Drivability stark eingeschränkt. Solche Straßenräume gibt es vor allem in historisch gewachsenen, dicht bevölkerten und vielseitig genutzten Stadtstrukturen. Im Gegensatz dazu sind weitläufigere Straßen, die vom Kfz dominiert und von anderen Verkehrsteilnehmenden kaum genutzt werden, deutlich besser für avF geeignet. Diese Art von Straßen gibt es vor allem in den Außenbezirken und im Umland. Zurzeit befinden sich auf diesen Straßentypen eher weniger Radfahrende. Beispiele für diese Straßentypologien sind Gewerbe-, Industrie-, Sammel- oder Einfahrstraßen. Diese Differenzierung ähnelt der von Soteropoulos erarbeiteten Drivability am Beispiel Wiens, in der aufgezeigt wird, dass ältere, meist zentral gelegene Quartiere eine größere Herausforderung für avF darstellen als periphere gelegene, autoaffine Stadtbereiche (Mitteregger et al. 2020: 81-84). Zusammenfassend lässt sich feststellen, dass sich avF in dichten innerstädtischen Quartieren mit einer hohen Anzahl Radfahrenden, wenn sich diese frei im Straßenraum bewegen, an deren Geschwindigkeit anpassen müssen.

\section{EIGNUNG VON RADVERKEHR-FÜHRUNGSFORMEN FÜR AVF: MISCHVERKEHR ODER GETRENNTER VERKEHR?}

Der Radverkehr wird in Deutschland hauptsächlich über vier Führungsformen in Straßenräume integriert: (A) ohne Infrastruktur frei im Straßenraum, auf markierten (B) Schutz- bzw. (C) Radfahrstreifen oder baulich getrennt bzw. (D) auf einem geschützten Radfahrstreifen (vgl. Abb. 4).

Abbildung 4: Führungsformen des Radverkehrs

A: ohne Infrastruktur

B: Schutzstreifen

C: Radfahrstreifen

D: geschützter Radfahrstreifen

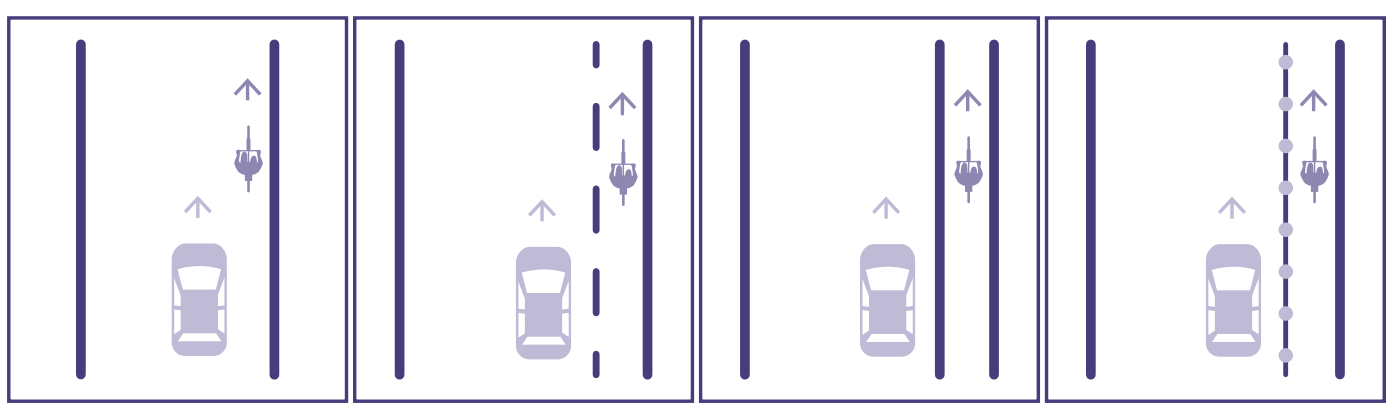

Quelle: eigene Darstellung 
Große Unterschiede in der Interaktion zwischen avF und Radfahrenden entstehen dabei nur, wenn der Radverkehr separat angelegt ist. Neben den unterschiedlichen Führungsformen haben auch unterschiedliche Straßenräume und Quartiere abweichende Eignungen für ein Miteinander von Radfahrenden und avF (vgl. vorheriger Abschnitt).

Die Vor- und Nachteile von einer getrennten Führungsform für Radfahrende werden von PlanerInnen und UnfallforscherInnen kontrovers diskutiert. Welche Auswirkungen die beiden Verkehrssysteme auf ein Miteinander von avF und Radfahrenden haben könnten, wird im folgenden Abschnitt skizziert (vgl. Tab. 2). Zunächst werden die Folgen einer Trennung von Rad- und motorisiertem Verkehr verdeutlicht. Beispiele für Städte mit einer verstärkten Trennung der Verkehrsarten finden sich in Dänemark und den Niederlanden (Gehl 2010: 213; Bernold et al. 2017; Milde 2017). Die meisten anderen westeuropäischen Länder haben Radfahrende größtenteils im Mischverkehr integriert. Städte wie Paris, Brüssel oder auch Berlin beginnen, den Radverkehr vermehrt getrennt auf eigenen Radfahrstreifen zu führen, insbesondere auf Hauptverkehrsstraßen.

Positiv an einer Trennung von Radverkehr und motorisiertem Verkehr ist für Radfahrende, dass die subjektive Sicherheit für sie steigt (NRVP 2019, FixMyCity 2020). Durch eine Erhöhung der Sicherheit können größere Teile der Bevölkerung zum Radfahren motiviert werden (Sinus 2017: 67, 70-72, 140), was ferner zu weiteren Sicherheitsgewinnen für Radfahrende führen kann. Die Unfallzahlen von Radfahrenden sind in Ländern mit hohem Anteil von Radverkehr deutlich niedriger. Dies wird auf den „Safety in Numbers“ bezeichneten Effekt zurückgeführt: Autofahrende, die mit Radfahrenden rechnen, erzeugen seltener Unfälle mit ihnen (Jacobsen 2003; Dutch Cycling 2019; Gehl 2010: 216; Woolsgrove/Armstrong 2020: 6; Greenpeace 2018: 10). Inwiefern dies auch auf avF zutrifft, ist noch unklar.

Bei Straßentypen, die von einer hohen Anzahl an unterschiedlichen Verkehrsteilnehmenden frequentiert werden, sind baulich getrennte Radwege für avF die einzige Möglichkeit, um autonom die zulässige Geschwindigkeit erreichen zu können (Taub 2019, Heinrichs 2015). Gründe hierfür sind die gesetzlichen Abstandsregeln beim Überholen sowie die Schwierigkeit für avF, Bewegungen von Radfahrenden vorauszusehen (Randelhoff 2017, Mingels 2019).

Getrennte Radwege sind in Deutschland innerstädtisch bisher kaum verbreitet, es müsste also großfächig neue Infrastruktur gebaut werden. Je nachdem, wie eine bauliche Trennung gestaltet wird, kann sie die Durchlässigkeit von Straßen, die Aufenthaltsqualität und Barrierefreiheit in Städten einschränken und damit die Umsetzung des Leitbildes der gemischten und lebendigen Stadt erschweren. Zusätzlich müssten der öffentliche Raum und der Verkehrsraum stark verändert werden. Neue Flächen für separierte Radwege können entstehen, indem Fahrspuren des ruhenden und fließenden motorisierten Verkehrs umgenutzt werden.

Vorteile des Mischverkehrs sind, dass die Infrastruktur und der öffentliche Raum wenig angepasst bzw. umgebaut werden müssen. Offene Straßenräume, in denen alle Verkehrsteilnehmenden gleichberechtigt aktiv sind und gegenseitig Rücksicht nehmen, spiegeln die Vielfalt der belebten europäischen Stadt wider. Sie ermöglichen Lebendigkeit und Offenheit, die lebenswerte Städte ausmachen und tragen damit zu einer hohen Lebensqualität in Städten bei. Im gemischten Verkehr müssen alle regelkonform fahrenden Verkehrsteilnehmenden ihre Geschwindigkeiten aneinander anpassen, was insgesamt deutlich niedrigere Geschwindigkeiten zur Folge hätte. Das Risiko von schweren oder tödlichen Unfällen sinkt bei langsamerem Verkehr drastisch. Die Stadt Oslo, die speziell ihren motorisierten Verkehr verlangsamt und zusätzlich getrennte Infrastruktur für Radfahrende an Hauptstraßen zur Verfügung stellt, registrierte im Jahr 2019 keine im Verkehr tödlich verunglückte Radfahrende und Zufußgehende (Bliss 2018, Walker 2020). 
Für avF sind gemischte Verkehrssituationen eine große Herausforderung, da sie Probleme haben, auf das intuitive Fahren der fahrergeführten Fahrzeuge und der Radfahrenden im offenen Straßenraum zu reagieren. Die defensive Fahrweise von Radfahrenden wird sie häufig ausbremsen und ein Überholen ist, unter Wahrung der gesetzlich vorgeschriebenen Mindestabstände, selten möglich. Für einen regelkonformen Überholvorgang von Radfahrenden ist entweder eine zweite Fahrspur oder eine einzelne Fahrspur mit 5,5 Metern Breite erforderlich (vgl. FGSV 2006: 27, Abschnitt rechtliche Einordnung Radverkehr). Bei der Analyse von bestehenden Straßen und den Empfehlungen aus den RASt 06 wird klar, dass das bestehende Straßennetz ein regelkonformes Überholen häufig erschwert. Bei Gegenverkehr ist ein regelkonformes Überholen meistens ausgeschlossen (vgl. Tab. 1).

Tabelle 2: Radfahrende und automatisierte und vernetzte Fahrzeuge im Misch- oder getrennten Verkehr: Stärken und Schwächen

\begin{tabular}{lll}
\hline \multicolumn{3}{c}{ Mischverkehr } \\
\hline Stärken & Schwächen \\
\hline
\end{tabular}

- Keine neue Infrastruktur nötig

- "Offene" Straßenräume, die eine hohe Aufenthaltsqualität bieten.

- Alle regelkonform fahrenden Verkehrsteilnehmenden müssen langsamer fahren: Die Folge sind weniger schwerwiegende Unfälle und verstärkte Potentiale von aktiver Mobilität.

- Gegenseitige Rücksichtnahme ist Voraussetzung und sorgt für einen Verkehr auf Augenhöhe.
- Große Probleme für avF, im städtischen Verkehr zu fahren.

- Hoch- und vollautomatisierte Fahrzeuge müssen wahrscheinlich in begrenzten Straßenräumen mit Mischverkehr in den manuellen Modus wechseln oder Schrittgeschwindigkeit fahren.

- Niedrige subjektive Sicherheit bei Radfahrenden, die aber bei hohen avF-Anteilen steigen kann, da diese regelkonform fahren und überholen.

- Regelkonformes Überholen von Radfahrerinnen und Radfahrern ist auf den meisten innerstädtischen Straßen Deutschlands nicht oder nur schwer möglich.

\section{Getrennter Verkehr}

\section{Stärken}

Schwächen
- AvF haben weniger Probleme, mit Verkehrsteilnehmenden zu interagieren.

- Hohe subjektive Sicherheit für Radfahrende

- Mehr Menschen werden motiviert, Rad zu fahren. Dies kann die Sicherheit für alle Radfahrenden erhöhen.
- Großflächiger Bau von neuer Infrastruktur nötig

- Hoher Flächenverbrauch, in Straßen mit geringen Querschnitten nicht möglich

- Je nach Gestaltung der Trennungen zwischen den Verkehrsarten wird die Barrierefreiheit in Städten eingeschränkt. 
Basierend auf dem derzeitigen Forschungsstand zu den Vor- und Nachtteilen von getrennter bzw. gemischter Infrastruktur können unterschiedliche Straßentypen gezielt entwickelt werden, um einen effizienten Verkehr für lebenswerte Städte zu ermöglichen. Separierte Radinfrastruktur ist an relativ breiten Hauptverkehrsstraßen die beste Möglichkeit, insbesondere dann, wenn motorisierte Verkehrsteilnehmende schneller als $30 \mathrm{~km} / \mathrm{h}$ fahren. Die Flächen für diese Infrastruktur sollten, soweit es möglich ist, aus denen des ruhenden und fahrenden motorisierten Verkehrs gewonnen werden. Ein Mischverkehr ist auf Nebenverkehrsstraßen zielführend, wenn die Geschwindigkeit von motorisierten Fahrzeugen auf maximal $30 \mathrm{~km} / \mathrm{h}$ beschränkt ist. Dies würde bei einem steigenden Anteil von Radfahrenden dazu führen, dass avF ihre Geschwindigkeit wegen fehlenden Überholmöglichkeiten an Radfahrende anpassen, was den gesamten Verkehr sicherer macht.

\section{DIE BEDEUTUNG VON KNOTENPUNKTEN}

Knotenpunkte sind häufig Orte der Begegnung mit einer starken Bedeutung für das Stadtleben. Gleichzeitig sind sie Unfallschwerpunkte, speziell zwischen Radfahrenden und motorisiertem Verkehr. Gestaltungsvarianten wie eine geschützte Kreuzung ${ }^{4}$ oder Kreuzungen, an denen Radfahrende durch dem MIV vorgelagerte Haltelinien und eine frühere Grünphase besser sichtbar sind, sollen Unfälle verhindern. Da die Sensoren von avF so angebracht sind, dass keine toten Winkel entstehen, könnten viele Unfälle verhindert werden. Genauso wie fahrergeführte Fahrzeuge benötigen avF ein freies Sichtfeld, um Radfahrende unter allen Bedingungen detektieren zu können. Aus diesem Grund empfiehlt es sich, auch bei avF das Parken an Knotenpunkten zu verhindern und stattdessen Nutzungen zu finden, welche die freie Sicht auf den gesamten Knotenpunkt für alle Verkehrsteilnehmenden ermöglichen. Des Weiteren könnten avF die Fahrweise von Radfahrenden besser berechnen, wenn die Möglichkeiten ihrer Fahrweise begrenzt sind. Knotenpunkte, an denen indirektes Linksabbiegen gefördert wird (vgl. Abb. 5) oder Fahrradinfrastrukturen plötzlich enden, erschweren es, die Bewegungen von Radfahrenden vorauszusehen.

Abbildung 5: Kreuzung mit Führungen für indirektes Linksabbiegen

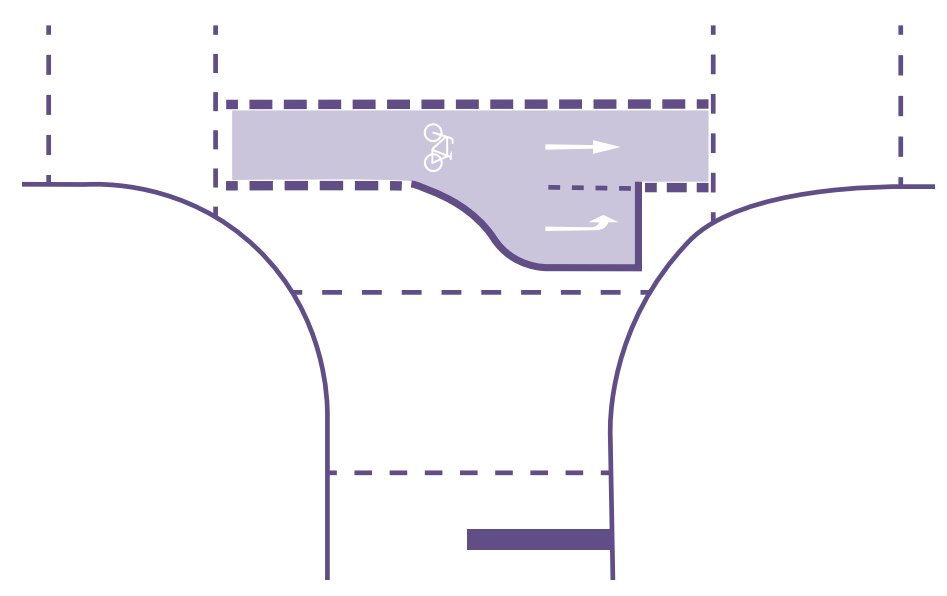

Quelle: eigene Darstellung nach RASt 06 (FGSV 2006)

$4 \quad$ Eine Kreuzung, bei der Radfahrende und Fußgängerlnnen vom Kraftverkehr getrennt sind und auf zurückgesetzten separierten Fahrstreifen queren (vgl. Darmstadt fährt Rad 2020). 


\section{FAZIT}

Radfahrende sind agil, wendig und haben spezielle Verkehrsregeln. Die nicht erst seit der Corona-Pandemie stark wachsenden Anteile von Radfahrenden stellen die avF vor dementsprechend große Herausforderungen. Die ersten Teilergebnisse der Arbeitspakete des Lehrstuhls Stadtplanung im Forschungsprojekt „Konzepte zur Integration des Radverkehrs in zukünftige urbane Verkehrsstrukturen mit autonomen Fahrzeugen - RAD-AUTO-NOM“ zeigen, dass sich avF in dichten Quartieren, die von vielen Radfahrenden frequentiert werden, ohne Anpassungen der Infrastruktur oder der Geschwindigkeit nur schwer bewegen werden können.

Die steigenden Unfallraten von Radfahrenden mit viel zu häufigen tödlichen Folgen zeigen, dass ein hoher Handlungsbedarf besteht, den städtischen Verkehrsraum sicherer zu gestalten. Hier besteht eine große Chance, dass avF den Verkehr gefahrloser machen. Im Gegensatz zu fahrergeführten $\mathrm{Kfz}$ werden ihre Systeme eine absolute Regeltreue durchsetzen und große Sicherheitsabstände zu Radfahrenden einhalten. Das kann den Großteil von Unfällen, für den die Pkw derzeit überwiegend die Hauptschuld haben, verhindern. Eine Reduzierung von haltenden und parkenden Kfz kann Gleiches bewirken: Ein Fünftel aller Radfahrunfälle stehen mit parkenden $\mathrm{Kfz}$ in Verbindung.

Die Regelakzeptanz von Radfahrenden wird bei für sie angepasster Infrastruktur besser. Dies ist speziell für einen Verkehr mit avF wichtig, da die fehlende Regelakzeptanz zu einem zentralen Konfliktpunkt für den durchregulierten und kontrollierten automatisierten Fahrverkehr werden kann (intuitives Fahren versus regulierte und intolerante Systeme).

Das städtische Umfeld und die Gestaltung des Straßen- bzw. Öffentlichen Raums hat einen großen Einfluss auf die Lebensqualität in Städten und die Sicherheit ihrer Verkehrsteilnehmenden. Dichte und kompakte Städte können einen hohen Anteil des MIV-Verkehrs vermeiden und mehr geteilt genutzte Flächen im öffentlichen Raum schaffen. Verstärkt durch die CoronaPandemie wird die städtische Mobilität weltweit neu gedacht. Der Straßenraum wird als „urban terrace“ gesehen, als ein großer „shared space“, in dem sich Gastronomie, Warteschlangen und große Menschenmengen mindestens gleichberechtigt zum Autoverkehr aufhalten können. Dieser Umbau kann nur gelingen, wenn in Städten mehr Flächengerechtigkeit herrscht und nicht mehr die größten Teile der Verkehrsfläche für den MIV genutzt werden. Allein die Flächen für den ruhenden Verkehr würden in vielen Straßenräumen ausreichen, um adäquate Flächen für mehr aktive Mobilität zur Verfügung zu stellen.

Fahrradfreundliche Städte in Dänemark und den Niederlanden, die als europäische Fahrradvorreiter gelten, sind besser für einen zukünftigen Verkehr von Fahrrad und avF ausgerichtet. Durch die teilweise getrennte und speziell für Radfahrende gebaute Infrastruktur ist die Regelakzeptanz von Radfahrenden höher, wodurch ihr Fahrverhalten für avF leichter vorhersehbar sein könnte.

Eine weitere Möglichkeit, Radfahren berechenbarer zu gestalten, besteht darin, Straßen so anzupassen, dass die gesetzlich geforderten Überholabstände zu Radfahrenden eingehalten werden können. Zusätzlich können Barrieren für den Rad- und Fußverkehr ein Überqueren an ausgewählten Straßen erschweren. Solche Maßnahmen würden die Straßenräume allerdings stark beeinträchtigen und die Städte deutlich weniger lebenswert und vielfältig machen. Dies würde den „[g]rundlegende[n] Widerspruch: öffentliche Räume hoher Aufenthaltsqualität und avM bei höheren Geschwindigkeiten“ (Mitteregger et al. 2020: 138) verwirklichen und eine neue autogerechte Stadt darstellen. 
Auch wenn es Leitziele der Verkehrspolitik Deutschlands zu Radfahrenden und auch zu avM gibt, hängt es stark von künftigen gesetzlichen Regelungen ab, wie die Städte an avF und die sich generell verändernden Rahmenbedingungen von Verkehr angepasst werden müssen. Ein für StadtplanerInnen wichtiges Ziel ist es, „Inseln der Autonomie“ - Bereiche oder Straßen, in denen es früher möglich ist, automatisiert zu fahren - nicht ungeplant entstehen zu lassen. Ziel sollte es sein, den geplanten Einsatz von avF zum Anlass zu nehmen, die Straßenräume für alle Verkehrsteilnehmenden gleichberechtigt zu gestalten, insbesondere auch in Siedlungen am Stadtrand. Dabei ist auch ein automatisierter ÖV zu berücksichtigen. Geringere Höchstgeschwindigkeiten im MIV und mehr Raum für Verkehrsteilnehmende, die einen geringen Flächenverbrauch haben (ÖPNV, Radfahrende, FußgängerInnen und Elektrokleinstfahrzeuge), sind wichtige Maßnahmen für lebenswerte Stadtquartiere mit öffentlichen Räumen für alle Teile der Bevölkerung.

Ein wachsender Radverkehr und die schrittweise Einführung von automatisierter und vernetzter Mobilität verändert wenig Grundlegendes an den gängigen Gestaltungsschwerpunkten für lebendige Städte mit sicheren Verkehrsräumen. Zu nennende Leitlinien sind u. a.:

- Freihalten von Sichträumen für alle Verkehrsteilnehmenden,

- ausreichend Flächen für die unterschiedlichen Verkehrsarten, auf denen mit niedrigen Geschwindigkeiten gefahren wird, und

- eine kompakte, funktionsgemischte Stadtstruktur, die den Verkehr reduziert.

Um automatisiertes Fahren in urbanen Räumen mit hohem Radverkehrsanteil zu ermöglichen, sollten in der Regel die Höchstgeschwindigkeiten an Radfahrende angepasst werden. Dies gilt primär für enge Straßenräume mit vielen unterschiedlichen Verkehrsteilnehmenden. Im Rahmen der Mobilitätswende und der Corona-Pandemie sind bereits Geschwindigkeitsreduzierungen in einigen Innenstädten zu beobachten (z. B. Brüssel, Mainz oder Bilbao). Somit könnte der gesamte öffentliche Raum als „urban terrace“ und „shared space“ genutzt und Unfälle stark reduziert werden.

\section{AUSBLICK UND FORSCHUNGSBEDARF}

Im weiteren Verlauf des Forschungsprojekts werden konkrete Empfehlungen und Gestaltungsansätze zur Konfliktvermeidung zwischen Radfahrenden und avF erarbeitet. Dazu führt das Institut für Mobilität und Verkehr (imove) der Technischen Universität Kaiserslautern Befragungen und Fahrversuche durch, um mit verkehrswissenschaftlichen Methoden die Belange der funktionalen Sicherheit zu ermitteln. Das Fachgebiet Elektromobilität errichtet eine Simulationsumgebung zur Bewertung der Wechselwirkungen zwischen verkehrlich-planerischen Konzepten und technischen Systemen. Dafür werden Bewegungsprofile von Radfahrenden mit Methoden des maschinellen Lernens ausgewertet, um eine Erkennung und Vorhersage zu ermöglichen. Der Lehrstuhl Stadtplanung wird stadtplanerische Konzepte zur Gestaltung von Radverkehrsanlagen im öffentlichen Raum unter avF-Bedingungen erstellen.

Die Frage, wie Städte planerisch mit steigenden Anteilen von avF umgehen, bietet vielfältigen zusätzlichen Forschungsbedarf: Im vorliegenden Beitrag ist wenig auf die zunehmende Ausdifferenzierung von Mikromobilität (Radfahrende, Elektrokleinstfahrzeuge, Pedelecs, Lastenfahrzeuge und Ähnliches) und den Einfluss dieses Trends auf avF eingegangen worden. Des Weiteren sind Knotenpunkte von enormer Bedeutung für einen konfliktfreien Verkehr. Gerade auch 
wegen ihrer stadträumlichen und verkehrlichen Bedeutung sollten sie differenziert untersucht werden. Dabei sind Unterschiede der automatisierten Drivability, je nach Art des Knotenpunktes, eine wichtige Frage. Grundsätzlich ist zu diskutieren, wie die Level des automatisierten Fahrens stärker auf unterschiedliche Straßenräume und das Miteinander von unterschiedlichen Verkehrsarten angepasst werden können (vgl. Stayton/Stilgoe 2020). Dies würde helfen zu planen, wo und wie avF fahren können.

\section{LITERATUR}

ADAC 2019. „Digitale Hilfe für den Schulterblick Ausstiegswarner gegen Dooring-Unfälle im Vergleich“, 20.8.2019. München. https://tinyurl.com/yuanftyc (9.10.2019).

ADFC (Allgemeiner Deutscher Fahrrad-Club) 2018a. „Verkehrsrechte für Radfahrende“, 28.4.2020. Berlin. https://tinyurl.com/5xzzbs7r (3.9.2019).

ADFC 2018b. „Sichere Kreuzungen durch mehr Fahrradstellplätze“, 24.4.2018. Berlin. https://tinyurl. com/jfet72f8 (18.9.2019).

ADFC 2018c. Positionspapier geschützte Radfahrstreifen, 13.4.2018. Berlin. https://tinyurl.com/6hzva7du (20.6.2020).

ADFC 2019. „So geht Verkehrswende - Infrastrukturelemente für den Radverkehr“, Mai 2019. Berlin. https://tinyurl.com/jyfbr9tt (9.10.2019).

Agentur für clevere Städte (Hrsg.) 2014. „Wem gehört die Stadt? Der Flächen-Gerechtigkeits-Report“, 5.8.2014. Berlin. https://tinyurl.com/rmttt8 (28.12.2019).

Alessandrini, A., A. Campagna, P. D. Site, F. Filippi und L. Persia 2015. „Automated vehicles and the rethinking of mobility and cities", in Transportation Research Procedia 5, 145-160.

Alrutz, D., W. Bohle, H. Müller und H. Prahlow 2009. Unfallrisiko und Regelakzeptanz von Fahrradfahrern. Bergisch Gladbach: Wirtschaftsverlag NW.

Amelang, S. 2020. "Corona crisis shakes up shift to sustainable urban mobility", Clean Energy Wire, 29.5.2020. www.cleanenergywire.org/news/corona-crisis-shakes-shift-sustainable-urban-mobility (4.6.2020).

Awad, E., S. Dsouza, R. Kim. J. Schulz, J. Heinrich, A. Shariff, J. Bonnefon und I. Rahwan 2018. „The Moral Machine experiment", in Nature 563, 59-64.

Beckmann, K. J., und G. Sammer 2016. „Autonomes Fahren im Stadt-und Regionalverkehr, Memorandum für eine nachhaltige Mobilitätsentwicklung aus der integrierten Sicht der Verkehrswissenschaft", 25.10.2016. DOI: 10.13140/RG.2.2.21205.42721.

Bernold, M., M. Pintner und A. Felczak 2017. „Niederlande: Erfolgsgeheimnisse aus dem Fahrrad-Paradies“, in Drahtesel. Das österreichische Fahrradmagazin 3, 9-11. https://tinyurl.com/6k3c5ntt (6.10.2020).

Bliss, L. 2018. „The War on Cars, Norwegian Edition“, Bloomberg CityLab, 3.5.2018. www.citylab.com/ transportation/2018/05/oslos-race-to-become-a-major-bike-haven/559358/ (4.1.2020).

BMVI (Bundesministerium für Verkehr und digitale Infrastruktur) 2012. „Nationaler Radverkehrsplan 2020: Den Radverkehr gemeinsam weiterentwickeln“. Berlin. https://tinyurl.com/2uj8nkw2 (30.8.2019).

BMVI 2015. „Automatisiertes und vernetztes Fahren“. Berlin. https://tinyurl.com/7xw8atmj (18.11.2019).

BMVI 2019a. „Bundesministerium für Verkehr und digitale Infrastruktur: Fahrradverkehr - Radfahren schützt das Klima und die Umwelt". Berlin. https://tinyurl.com/sjpxzzzt (19.9.2019).

BMVI 2019b. „Die acht Leitziele des nationalen Radverkehrsplans“. Berlin. www.zukunft-radverkehr. bmvi.de/bmvi/de/home/info/id/15 (19.9.2019).

BMVI 2020. „Wir machen den Straßenverkehr noch sicherer, klimafreundlicher und gerechter“. Berlin. www.bmvi.de/SharedDocs/DE/Artikel/K/stvo-novelle-sachinformationen.html (19.6.2020).

Bonnefon, J., A Shariff und I. Rahwan 2016. „The social dilemma of autonomous vehicles“, in Science 6293, 1573-1576. 
Botello, B., R. Buehler, S. Hankey, A. Mondschein und Z. Jiang 2019. „Planning for walking and cycling in an autonomous-vehicle future", in Transportation Research Interdisciplinary Perspectives 1, 1-4. DOI: 10.1016/j.trip.2019.100012.

Burger, J. 2019. „Radfahren: Neben der Spur“, in Zeitmagazin 26, 18.9.2019, 14-20.

Copenhagenize.com 2017. „Arrogance of Space - Copenhagen - Hans Christian Andersen Boulevard“, 9.5.2017. https://tinyurl.com/2az63v42 (2.1.2020).

Dangschat, R. 2018. „Automatisierung des (urbanen) Verkehrs - Neu-Erfindung oder Widerspruch zur ,Europäischen Stadt'“, in Urbanität im 21. Jahrhundert, hg. N. Gestring und J. Wehrheim, Frankfurt am Main/New York: Campus, 313-335.

Darmstadt fährt Rad 2020. „Wunderlösung Schutzkreuzung? - Teil 2“, 19.1.2020. www.darmstadtfaehrtrad.org/?p=2594 (22.1.2020).

Destatis (Statistisches Bundesamt) 2019. „Verkehrsunfälle: Kraftrad- und Fahrradunfälle im Straßenverkehr 2018“, 19.8.2019. Wiesbaden. https://tinyurl.com/y2kn3a7c (20.9.2019).

Dickmanns, E. 2020. „Dickmanns Pionierarbeit zum autonomen Fahren“, Presseinformation, 28.2.2020, Karlsruhe: Fraunhofer-Institut für Techno- und Wirtschaftsmathematik ITWM. https://tinyurl.com/ pm2284pv (4.6.2020).

Dill, J. 2015. „Webinar, Part II: Four Types of Cyclists: A National Look“, TREC Webinar Series, Book 5, 11.8.2015. www.core.ac.uk/download/pdf/81253510.pdf (19.9.2019).

DLR 2015. „XCYCLE Projekt - Advanced Measures to Reduce Cyclists' Fatalities and Increase Comfort in the Interaction with Motorised Vehicles“, funded by The Horizon 2020 Framework Programme of the European Union, Universita di Bologna. www.xcycle-h2020.eu/, www.cordis.europa.eu/project/id/635975 (12.7.2020).

Dutch Cycling 2019. „Die Niederlande - Eine Entwicklungsgeschichte des Radfahrens“, The Dutch Cycling Embassy. www.dutchcycling.nl/images/downloads/Brochure_German.pdf (10.10.2019).

DVR (Deutscher Verkehrssicherheitsrat) 2019. „Dooring-Unfälle: Mehr als jeder zehnte Auto Fahrende beim Aussteigen unaufmerksam“, 9.9.2019. Bonn. https://tinyurl.com/6xbz7x46 (9.10.2019).

FGSV (Forschungsgesellschaft für Straßen- und Verkehrswesen) 2006. „Richtlinien für die Anlage von Stadtstraßen - RASt 06“, Ausgabe 2006. Download unter https://tinyurl.com/3j2jdvsx (19.8.2020).

FixMyCity 2020. „Studie zur subjektiven Sicherheit im Radverkehr - Ergebnisse und Datensatz einer Umfrage mit über 21.000 Teilnehmenden“, 6.7.2020. Berlin. www.fixmyberlin.de/research/subjektive-sicherheit (10.7.2020).

Fleming S. 2017. Velotopia: The Production of Cyclespace in Our Minds and Our Cities. Rotterdam: nai010.

Francke, A., J. Anke und S. Lißner, 2018. „Sag mir, wie du radelst und ich sage dir, welche Infrastruktur du dir wünschst - Darstellung erster Ergebnisse einer Radfahrtypologie“, in 26. Verkehrswissenschaftliche Tage: Grenzenlos(er) Verkehr!?, Tagungsband, hg. v. TU Dresden, 139-152.

GDV (Gesamtverband der Deutschen Versicherungswirtschaft) 2015. „Geschwindigkeitswahrnehmung von einspurigen Fahrzeugen“, 12.6.2015. Berlin. Download unter www.udv.de/de/publikationen/forschungsberichte/geschwindigkeitswahrnehmung-einspurigen-fahrzeugen (1.7.2020).

GDV 2016. „Typische Unfälle zwischen Pkw und Radfahrern“. Berlin. Download unter www.udv.de/ download/file/fid/10069 (1.4.2020).

GDV 2020. „Unfallrisiko Parken für Fußgänger und Radfahrer“, Unfallforschung kompakt Nr. 98. Berlin. Download unter https://udv.de/download/file/fid/12576 (12.7.2020).

Gehl, J. 2010. Städte für Menschen, 3. Auflage. Berlin: Jovis.

Geller, R. 2009. „Four Types of Cyclists“. Portland, OR: Portland Bureau of Transportation. www.portlandoregon.gov/transportation/article/158497 (9.5.2019).

Greenpeace 2018. „Radfahrende schützen - Klimaschutz stärken: Sichere und attraktive Wege für mehr Radverkehr in Städten“, 1.8.2018. Hamburg. https://tinyurl.com/fwfp7jy8 (10.2.2020).

Heinrichs, D. 2015. Autonomes Fahren und Stadtstruktur. Berlin/Heidelberg: Springer Vieweg.

Heinrichs, D. 2017. „Autonomes Fahren fordert die Planung heraus“, Treffpunkt Kommune, 5.10.2017. www.treffpunkt-kommune.de/autonomes-fahren-fordert-die-planung-heraus/ (20.9.2019).

Huemer, A., und K. Eckhardt-Lieberam 2016. „Regelkenntnisse bei deutschen Radfahrerlnnen: Onlinebefragung unter Erwachsenen und SchülerInnen“, in Zeitschrift für Verkehrssicherheit (62) 5, 21-27. 
ILS (Institut für Landes- und Stadtentwicklungsforschung) 2013. „Einstellungsorientierte Akzeptanzanalyse zur Elektromobilität im Fahrradverkehr“, ILS-Forschung 1/2013. Dortmund. www.repository.difu.de/jspui/bitstream/difu/220522/1/DM13110843.pdf (10.7.2020).

ISI (Fraunhofer-Institut für System- und Innovationsforschung ) 2019. „Energie- und Treibhausgaswirkungen des automatisierten und vernetzten Fahrens im Straßenverkehr", Wissenschaftliche Beratung des BMVI zur Mobilitäts- und Kraftstoffstrategie. Karlsruhe. https://tinyurl.com/ u587csvs (15.12.2019).

Jacobsen, P. 2003. „Safety in numbers: more walkers and bicyclists, safer walking and bicycling“, in Injury Prevention (9) 3, 205-209.

Kahn, J. 2018. „To Get Ready for Robot Driving, Some Want to Reprogram Pedestrians“, Bloomberg News, 16.8.2018. https://tinyurl.com/ymajj9vv (15.9.2020).

Klein, T., D. Köhler, T. Stein und E. Süselbeck 2020. „Radverkehr im Ausnahmezustand. Mit Rückenwind aus der Krise?“, difu Deutsches Institut für Urbanistik, 2.6.2020. www.difu.de/nachricht/ radverkehr-im-ausnahmezustand-mit-rueckenwind-aus-der-krise (4.6.2020).

LAB (The League of American Bicyclists) 2014. „Bicyclists safety must be a priority: Findings from a year of fatality tracking - and the urgent need for better data“. Washington, DC. www.bikeleague. org/sites/default/files/EBC_report_final.pdf (10.6.2020).

Lee, P., J. Loucks, D. Stewart, G. Jarvis und C. Arkenberg 2019. „Technology, Media, and Telecommunications Predictions 2020“, Deloitte.Insights. https://tinyurl.com/24f5dh9e (2.1.2020).

Lemmer, K. 2019. Neue autoMobilität II: Kooperativer Straßenverkehr und intelligente Verkehrssteuerung für die Mobilität der Zukunft. München: Utz.

Louen, C. 2020. „Veränderungspotential im Straßenraum durch autonomes Fahren“, Vortrag bei der Tagung Autonomes Fahren und Stadtentwicklung, Kassel.

Milde, M. 2017. „Radverkehr in den Niederlanden und Deutschland. Ein kritisch-optimistischer Vergleich“, Vortrag, AGFS-Kongress 2017: 200 Jahre Fahrrad. https://tinyurl.com/2tw8fauy (6.10.2019).

Millard-Ball, A. 2019. „The autonomous vehicle parking problem“, in Transport Policy 75, 99-108. https://tinyurl.com/wm4a8ypy (7.11.2019).

Mingels, G. 2019. „Das Auto darf selbst fahren - so, wie ich es will“, spiegel.de, 1.11.2019, www. spiegel.de/plus/alex-roy-der-schrillste-kritiker-der-selbstfahrenden-autos-a-00000000-00020001-0000-000166735193 (7.11.2019).

Mitteregger, M., E. M. Bruck, A. Soteropoulos, A. Stickler, M. Berger, J. S. Dangschat, R. Scheuvens und I. Banerjee 2020. AVENUE21. Automatisierter und vernetzter Verkehr: Entwicklungen des urbanen Europa. Berlin: Springer Vieweg.

Neufert, E. 2009. Bauentwurfs/ehre. Wiesbaden: Vieweg+Teubner.

Nobis, C. 2019. „Mobilität in Deutschland - MID. Analysen zum Radverkehr und Fußverkehr“, Studie von infas, DLR, IVT und infas $360 \mathrm{im}$ Auftrag des Bundesministeriums für Verkehr und digitale Infrastruktur (FE-Nr. 70.904/15), Bonn/Berlin. https://tinyurl.com/3psam2vk (5.1.2020).

NPM (Nationale Plattform Zukunft der Mobilität) 2019. „Zweiter Zwischenbericht: Handlungsempfehlungen zum Autonomen Fahren. Arbeitsgruppe 3: Digitalisierung für den Mobilitätssektor“. Berlin. https://tinyurl.com/k9kuxdh5 (7.12. 2019).

NRVP (Nationaler Radverkehrsplan) 2019. „Keine Angst beim Radfahren: Subjektive Sicherheit im Radverkehr", Fahrradportal, 5.6.2019. Berlin: Bundesministerium für Verkehr und digitale Infrastruktur. www.nrvp.de/21242 (5.6.2019).

Randelhoff, M. 2017. „Zukunftsszenarien für das automatisierte Fahren in der Stadt - Chancen und Risiken für den Radverkehr“, Vortrag, 27.12.2017. www.zukunft-mobilitaet.net/166701/verkehrssicherheit/automatisiertes-fahren-radverkehr-zukunftsszenarien-stadtverkehr-vortrag/ (18.9.2019).

Reid, C. 2018. „Cyclists Don't Respect Rules, Said Nissan CEO Carlos Ghosn Arrested On Suspicion Of Fraud“, Forbes, 19.11.2018. https://tinyurl.com/32fu2k5d (15.9.2019).

Richter, T., O. Beyer, J. Ortlepp und M. Schreiber 2019. „Sicherheit und Nutzbarkeit markierter Radverkehrsführungen“, Forschungsbericht Nr. 59. Berlin: Gesamtverband der Deutschen Versicherungswirtschaft. Download unter www.udv.de/download/file/fid/11903 (10.10.2019).

Ritz, J. 2018. Mobilitätswende - autonome Autos erobern unsere Straßen: Ressourcenverbrauch, Ökonomie und Sicherheit. Wiesbaden: Springer. 
Rothfuchs, K., und P. Engler 2018. „Auswirkungen des autonomen Fahrens aus Sicht der Verkehrsplanung“, in Straßenverkehrstechnik 8, 564-571.

SAE International 2018. „Taxonomy and Definitions for Terms Related to Driving Automation Systems for On-Road Motor Vehicles - J3016“, 15.6.2018. www.sae.org/standards/content/j3016_201806/ (20.4.2020).

Schreck, B. 2016. „Radverkehr - Unfallgeschehen und Stand der Forschung“, in Zeitschrift für Verkehrssicherheit (62) 2, 63-77.

Schreiber, M., und O. Beyer 2019. „Sicherheit und Nutzbarkeit markierter Radverkehrsführungen“, in Straßenverkehrstechnik (12), 585-865.

Schwab, A. 2019. „Radwege und niederländische Kreuzungen: Keine Wunderlösung“, in mobilogisch! $2,18-23$.

Seibert, S. 2020. „Gestärkt aus der Krise, gemeinsam die Mobilität der Zukunft gestalten“, Pressemitteilung 316, 8.9.2020. Berlin: Presse- und Informationsamt der Bundesregierung (BPA). https:// tinyurl.com/hv4vctxd (10.9.2020).

Sinus 2017. „Fahrrad-Monitor Deutschland 2017: Ergebnisse einer repräsentativen Online-Umfrage“, 25.10.2017. Heidelberg/Berlin: Sinus Markt- und Sozialforschung. https://tinyurl.com/way95epp (18.2.2020).

Skinner, R., und N. Bidwell 2016. „Making Better Places: Autonomous vehicles and future opportunities“, WSP I Parsons Brinckerhoff in association with Farrells. www.wsp.com/-/media/Sector/ Global/Document/Making-better-places.pdf (6.10.2020).

Soteropoulos, A., M. Mitteregger, M. Berger und J. Zwirchmayr 2020. „Automated drivability: Toward an assessment of the spatial deployment of level 4 automated vehicles“, in Transportation Research Part A: Policy and Practice 136, 64-84.

Stayton E., und J. Stilgoe 2020. „It's Time to Rethink Levels of Automation for Self-Driving Vehicles“, SSRN, 13.5.2020. Download unter https://tinyurl.com/4sfj6efa (7.10.2020).

Tagesspiegel 2019. „Radmesser“. https://interaktiv.tagesspiegel.de/radmesser/ (3.9.2019).

Taub, E. 2019. „How Jaywalking Could Jam Up the Era of Self-Driving Cars“, New York Times, 1.8.2019, www.nytimes.com/2019/08/01/business/self-driving-cars-jaywalking.html (6.10.2019).

Walker, A. 2020. „Oslo saw zero pedestrian and cyclist deaths in 2019. Here's how the city did it“, Curbed, 3.1.2020. https://tinyurl.com/56sy6yjk (7.1.2020).

Weert, C., und L. Ruhrort 2019. „Mobilitätsatlas: Daten und Fakten für die Verkehrswende “, hg. v. Heinrich-Böll-Stiftung und VCD Verkehrsclub Deutschland e. V. www.boell.de/sites/default/ files/2019-11/mobilitaetsatlas.pdf?dimension1=ds_mobilitaetsatlas2019 (6.11.2019).

Woolsgrove, C., und J. Armstrong 2020. „Best Practice Guide: Safer Cycling Advocate Program“. Brüssel: European Cyclists' Federation. https://tinyurl.com/53r5zn9p (15.1.2020).

Open Access Dieser Beitrag wird unter der Creative Commons Namensnennung 4.0 International Lizenz (http://creativecommons.org/licenses/by/4.0/deed.de) veröffentlicht, welche die Nutzung, Vervielfältigung, Bearbeitung, Verbreitung und Wiedergabe in jeglichem Medium und Format erlaubt, sofern Sie den/die ursprünglichen Autor(en) und die Quelle ordnungsgemäß nennen, einen Link zur Creative Commons Lizenz beifügen und angeben, ob Änderungen vorgenommen wurden.

Die in diesem Beitrag enthaltenen Bilder und sonstiges Drittmaterial unterliegen ebenfalls der genannten Creative Commons Lizenz, sofern sich aus der Abbildungslegende nichts anderes ergibt. Sofern das betreffende Material nicht unter der genannten Creative Commons Lizenz steht und die betreffende Handlung nicht nach gesetzlichen Vorschriften erlaubt ist, ist für die oben aufgeführten Weiterverwendungen des Materials die Einwilligung des jeweiligen Rechteinhabers einzuholen.

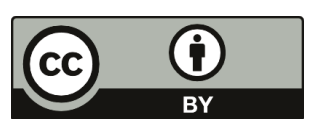

